\title{
Asthma control test to identify uncontrolled asthma in pediatric clinical practice
}

\section{To the Editor}

Asthma management is a daily challenge in pediatric practice as many clinical and functional aspects continuously change over time. Asthma management should be oriented to attain and maintain asthma control [1]. The asthma control level drives the intensity of care. Different tools can measure asthma control. The Global Initiative for Asthma (GINA) classification and the Asthma Control Test (ACT), and its pediatric version (childhood-ACT, C-ACT) are the most commonly used in clinical practice [1-3]. These two ways are popular in daily practice. Other instruments also are available, including the Composite Asthma Severity Index (CASI), which measures asthma severity [4]. Asthma severity is another facet relevant in the management. However, asthma control and severity are closely connected, and the concept of control/severity practically combines the two measures.

In this regard, Mokhallati et al. [5] evaluated the predictive value of CASI in a cohort of 108 children with asthma. ACT and C-ACT were also used to obtain additional information for contextualizing CASI. This study showed that CASI actually predicted the physician assessments of asthma control and severity and confirmed that both were not distinct entities. Moreover, ACT/C-ACT was a reliable predictor of physician-assessed control. Based on this background, we would evaluate the role of ACT/C-ACT in a large multicenter nationwide study. For this purpose, the Italian Society of Paediatric Allergy and Immunology promoted a perspectival study (“ControL'Asma") to investigate asthma control in children and adolescents managed in clinical practice. The first outcomes showed that the type
2 high allergic phenotype was prevalent, and partly controlled-uncontrolled asthma affected about half of the participants [6]. Further, the project also demonstrated that rhinitis is frequent asthma comorbidity and rhinitis phenotyping is useful for adequately managing asthma [7].

Consequently, the present study aimed to thoroughly evaluate the asthma control assessment in 469 children and adolescents (69.3\% males, mean age 11.2 years) consecutively visited across 10 Italian Paediatric Allergy centers. All patients were currently treated according to the GINA guidelines based on the asthma control level. The methodology has been reported in detail elsewhere [6].

The Ethics Committee of the Istituto Giannina Gaslini of Genoa initially approved the procedure (code number: 22253/2017; in the context of the Italian Project "ControL'Asma" promoted by the Italian Society of Paediatric Allergy and Immunology). All the other Review Ethics Committees further approved the study procedure and written informed consent was obtained from all parents. Clinical data were recorded by an electronic case report form designed expressly for this study.

Patient's characteristics at baseline were described as mean with standard deviation (SD), median with interquartile ranges (IQR), or count and percentage (\%), as appropriate. Univariable and multivariate logistic regression models were performed to evaluate factors associated with uncontrolled asthma. Variables with $\mathrm{p}<0.20$ in the univariate analysis were candidates for subsequent multivariate analysis. Receiver operating characteristic curve analysis was graphed to calculate the Area Under the Curve (AUC) and identify the optimal cut-off score for ACT to 
discriminate uncontrolled from controlled asthma (by calculating Youden's index). Two-sided P-values $\leq 0.05$ were considered statistically significant. The analyses were computed using SPSS Statistics version 21.0 (IBM Corp., Armonk, NY, USA).

According to GINA classification, $261(55.6 \%)$ subjects had well-controlled asthma, 152 (32.4\%) partly-controlled, and 56 (12\%) uncontrolled. At the multivariate analysis, only the adjusted age C-ACT/ACT (OR [odds ratio] $=0.66 ;$ CI [confidence interval] $0.59-0.75 ; \mathrm{p}$ $<0.001$ ) and forced volume vital capacity (OR $=0.95$; CI $0.91-0.98 ; \mathrm{p}=0.002$ ) were significantly associated with uncontrolled asthma. In particular, subjects with uncontrolled asthma had a C-ACT/ACT score of 17 (IQR 13-18), significantly $(\mathrm{p}<0.001)$ lower than subjects with controlled/partly-controlled asthma (23; IQR 20-25). The receiver operating characteristic curve identified a value of 18.5 as a reliable cut-off with AUC 0.875 (CI 0.83-0.91; p < 0.001 ), $87 \%$ sensitivity, $78.6 \%$ specificity, and 0.66 Youden Index, as reported in the Figure 1.

These outcomes are consistent with previous studies that provided robust evidence for the ACT as a reliable questionnaire to assess asthma control. In particular, an 18-month follow-up study demonstrated that C-ACT use improved asthma care [8]. Moreover, the ATS/ERS Task Force on asthma control concluded that the assessment should be multicomponent and consider clinical aspects using adequate tools, including the ACT [9]. A systematic review exploring the diagnostic performance of asthma control questionnaires concluded that ACT had low accuracy in identifying patients with uncontrolled asthma as the AUC was 0.69 [10]. Our outcomes were inconsistent with these findings. Namely, the ACT performance was good to define uncontrolled asthma correctly.

ACT is, therefore, a reliable and practical tool to precisely assess asthma control. Other tools, including CASI and asthma control questionnaire, need the lung function measurement. It yields the tests unusable in primary care settings, where spirometry is frequently unavailable. Moreover, in the COVID-19 pandemic era, telemedicine represents a promising alternative to office visits. Thus, careful history and ACT could represent an optimal way to manage asthma at home realistically.

On the other hand, the current study had some limitations, including the cross-sectional design and the lack of biomarker evaluation.

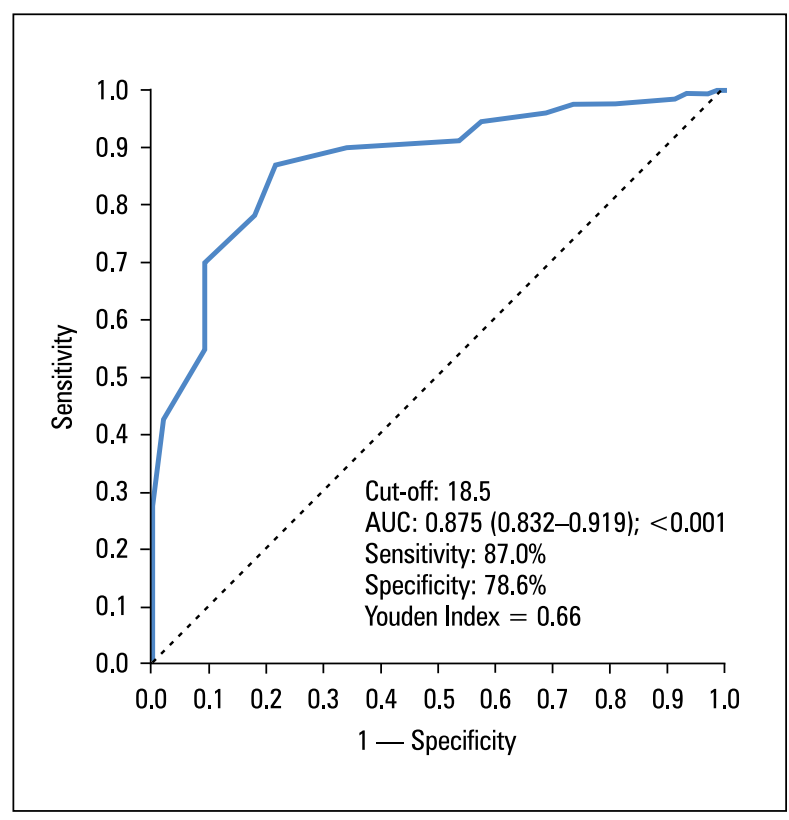

Figure 1. ROC curve for defining the optimal cut-off of C-ACT/ACT score in uncontrolled patients.

ACT — asthma control test; AUC — area under the curve; C-ACT — childhood-ACT; ROC — receiver operating characteristic

However, the study was conducted in a real-world setting, the number of participants was large, and the nationwide distribution of centers across Italy. These factors contributed to give outcomes that reflect what occurs in daily pediatric practice.

In conclusion, the ControL'Asma study showed that ACT is a suitable tool for identifying children and adolescents with uncontrolled asthma. As it is a questionnaire, ACT could be used in every setting, including primary care clinic and at home.

\section{Acknowledgments}

We would thank the members of the "ControL'Asma Study Group": Elisa Anastasio, Carlo Caffarelli, Federica Caricato, Loredana Chini, Elisabetta Del Duca, Valentina De Vittori, Maria Elisa Di Cicco, Ahmad Kantar, Maddalena Leone, Amelia Licari, Viviana Moschese, Diego G Peroni, Roberta Olcese, Michela Silvestri, Valeria Talarico, Martina Votto.

\section{Conflict of interest}

None declared.

\section{References:}

1. Global Initiative for Asthma. Global strategy for asthma management and prevention, 2021. www.ginasthma.org (17.03.2021). 
2. Nathan RA, Sorkness CA, Kosinski M, et al. Development of the asthma control test: a survey for assessing asthma control . J Allergy Clin Immunol. 2004; 113(1): 59-65, doi: 10.1016/j. jaci.2003.09.008, indexed in Pubmed: 14713908.

3. Liu AH, Zeiger RS, Sorkness CA, et al. Development and cross-sectional validation of the childhood asthma control test. J Allergy Clin Immunol. 2007; 119(4): 817-825, doi: 10.1016/j. jaci.2006.12.662, indexed in Pubmed: 17353040.

4. Wildfire JJ, Gergen PJ, Sorkness CA, et al. Development and validation of the Composite Asthma Severity Index--an outcome measure for use in children and adolescents. J Allergy Clin Immunol. 2012; 129(3): 694-701, doi: 10.1016/j. jaci.2011.12.962, indexed in Pubmed: 22244599.

5. Mokhallati N, Schuler CL, Thomas S, et al. Use of the composite asthma severity index in a pediatric subspecialty clinic. Ann Allergy Asthma Immunol. 2021; 126(6): 702-706, doi: 10.1016/j.anai.2021.01.036, indexed in Pubmed: 33549639.

6. Tosca MA, Marseglia GL, Ciprandi G, et al. "ControL'Asma" Study Group. The real-world „ControL'Asma” study: a nationwide taskforce on asthma control in children and adolescents. Allergol Immunopathol (Madr). 2021; 49(1): 32-39, doi 10.15586/aei.v49i1.14, indexed in Pubmed: 33528927.
7. Tosca MA, Duse M, Marseglia G, et al. "ControL’Asma” Study Group. The practical clinical relevance of rhinitis classification in children with asthma: outcomes of the „ControL'Asma” study. Ann Allergy Asthma Immunol. 2019; 123(5): 516-519, doi: 10.1016/j.anai.2019.08.003, indexed in Pubmed: 31401105.

8. Bousema S, Verwoerd AJ, Goossens LM, et al. Protocolled practice nurse-led care for children with asthma in primary care: protocol for a cluster randomised trial. BMJ Open. 2019; 9(9): e022922, doi: 10.1136/bmjopen-2018-022922, indexed in Pubmed: 31562140.

9. Reddel HK, Taylor DR, Bateman ED, et al. American Thoracic Society/European Respiratory Society Task Force on Asthma Control and Exacerbations. An official American Thoracic Society/European Respiratory Society statement: asthma control and exacerbations: standardizing endpoints for clinical asthma trials and clinical practice. Am J Respir Crit Care Med. 2009; 180(1): 59-99, doi: 10.1164/rccm.200801-060ST, indexed in Pubmed: 19535666

10. Jia CE, Zhang HP, Lv Y, et al. The asthma control test and asthma control questionnaire for assessing asthma control: systematic review and meta-analysis. J Allergy Clin Immunol. 2013 131(3): 695-703, doi: 10.1016/j.jaci.2012.08.023, indexed in Pubmed: 23058645. 